\title{
FROM THE RESEARCH OF ECONOMIC MOTIVATION AND METROLOGICAL BEHAVIOUR OF ECONOMIC ENTITIES TO THE RESEARCH OF METROLOGICAL SYSTEMS IN ECONOMICS AS A SPECIFIC TYPE OF SYSTEMS
}

\begin{abstract}
The basic historical types of economic motivation of economic entities as fundamentals of forming both the main historical types of metrological behavior and the most fundamental types of economic measurement are defined. A general characteristic of nominal, real and super-real economic measurements as the main subject of an economic metrology being a general theory of economic measurement is given. Based on the previous definition of the basic types of economic measurement a general characteristic of appropriate metrological systems in economics, namely, nominal, real and super-real ones is offered.
\end{abstract}

Key words: economic motivation, metrological behavior, economic measurement, nominal, real and super-real economic measurement, metrological systems, nominal, real and super-real metrological systems, economic metrology, metrological economy.

Introduction. The aim of this article is to carry out research of the general characteristics of metrological systems in economics on the basis of generalization of their historical and functional practice of formation as well as to analyze modern literature on these economic issues. The main idea of the article is to prove that the practice of economic measurements as well as of the metrological system, which is formed on its basis, is derived from, firstly, the economic motivation of economic entities and, secondly, the metrological behavior of these entities. According to the author, on the basis of his research, metrological systems in economics are determined by the nature of both economic behavior of economic entities and their metrological behavior. In this perspective, metrological systems in economics are studied for the first time in modern economic literature, unless, of course, we do not take into account the previous publications of the author on the above mentioned issues.

The main results of the study. The main result of the scientific analysis of the economic measurement practice carried out by the author is formation of the theoretical statement that the character, or nature, of economic measurement is determined by capital scales, the scales of economic class systems in which it is implemented. The larger really (just really, not nominally, as the author of this article notes) the system's scales are, the scales of certain capital, which always acts as a material-economic basis of these systems, the more perfect economic measurement is as well as an appropriate type of metrological systems being formed on its basis. The main goal of the scientific economic analysis is achieved in the plane of characterizing historical types of economic motivation of business entities, their metrological behavior and the most fundamental types of economic measurements. Such a research plane affected the structure of this article. First, the main historical types of economic motivation and metrological behavior of economic entities are analyzed, and later - the most fundamental types of economic measurements.

1. About the main historical types of economic entities economic motivation

In econometric studies of the latest (current or most modern) period we should not go from economic and mathematical models to the actual economic practice (and practice of economic measurement) but vice versa. Until now econometric studies have been done "upside down". First, there was constructed this or that economic and mathematical model using mathematical instruments without sufficient understanding of the real economic life, and later based on its mathematical (at best economic-mathematical) analysis some recommendations for economic practice were made. At this, economists-mathematicians (traditional econometrists) little thought about the principles of economic measurement used by a certain economic practice, about how it creates certain economic parameters. This essential economic problem remained outside econometric analysis. We are among the first in modern economic literature who attempted to move away from this style of econometric research. We put 


\section{G. I. Bashnyanyn}

it as the main goal to return to econometric researches their genuine economic substance and economic nature. Econometrics is primarily and mainly economic science, though it is also metrological science and only then it is or may be mathematical science (and even then only in strictly defined limits determined not by subjective intentions or desires of researchers, but by economic practice). To consider it otherwise is once again to turn it "upside down".

Study of principles, methods, rules or algorithms for forming and modifying the basic economic parameters should be based not on studying the various parts of economic and mathematical models (or economic and mathematical functions), as is still done in traditional or post-traditional branch (version) of econometric science (econometric research since its birth and in fact today has assigned great importance to partial derivatives of linear economic and mathematical models, considering it to be one of the forms of evaluating goods and services) but on studying the actual economic practice, especially its specific part or aspect - practice of economic measurement. It is in the practice of economic measurement that we should identify certain metrological procedures based on the principles or rules of economic parameters formation. The practice of economic measurement, as we noted in our previous metrological works, comprises two sectors. On the one hand, we can single out its market part (the practice of the so-called market pricing) and, on the other hand, there is its beyond-the- market part. However, common to both parts is the fact that they are forming economic parameters through a certain metrological activity of business entities, certain metrological behavior. Different economic entities in different economic conditions (historical and economic conditions) form economic parameters in different ways, and they do it before the market, a priori. In the compromisebased market (which is also determined by a number of factors) different approaches to economic measurement are only "agreed", and are reduced to a single compromise value. Therefore, metrological activity of businesses starts "before the market", among the major economic agents producers and consumers, and it just ends at the stage of the market and market structures.

This important circumstance of operation and development of the practice of economic measurement cannot be understood by liberal economists (to whom the majority of domestic reformers-practitioners of the national economy belong together with other economists from post-socialist countries).They think that the market mechanism performs the economic measurement procedure automatically and without any subjective activity of the main economic agents, at least, this concerns such a fundamental economic parameters as value. We follow a different perspective: the value is actually formed through the market mechanism, but through appropriate metrological procedures undertaken by major economic entities. Its content is usually an objective metrological form of final order (as it is formed by the economic entities with the account of certain factors), but at the same time it is a subjective form (as economic entities are "creating" it following strictly determined rules that correspond to their own interests and exactly reflect them). Economic entities "are placed" by an objective economic development in certain "metrological framework" and act within it. The main task of economic metrology as part of theoretical econometrics in a broad sense is to identify and theoretically fix these objective limits of subjective metrological activity of the major agents of a particular market or the economy as a whole. Therefore, we can definitely say that the main subject (or object if the latter is somewhat narrowly understood) of economic metrology (and econometrics in general) should be detecting and "decoding" of metrological behavior of economic entities because it is just through it that economic measurements are made. Such understanding of economic metrology subject was presented in our previous works. Being aware of the specifics of metrological researches in economics, we did not want to bring them down just to the economic analysis of economic and mathematical models or functions.

However, having defined the subject of economic metrology in this way, we have not answered the question: what factors objectively determine the metrological behavior of businesses? Why is it different in different economic situations? In some cases it is based on one type metrological principles, in others - on the other. Careful study of the practice of economic measurement and metrological behavior of businesses has given reasons for the fundamental conclusion that the metrological behavior of economic entities is determined by their economic motivation (this point of view is shared by I. M. Kopych who is one of major "developers" of general economic metrology as both theoretical and 
From the research of economic motivation and metrological behaviour of economic entities...

fundamental part of econometrics in a broad sense); with changing the nature of economic motivation the metrological behavior of economic entities is changing correspondingly. The first means the ultimate direction or the main purpose of a certain business activity. It gives the answer to the question why, for what purpose we carry out this or that economic activity (fig. 1).

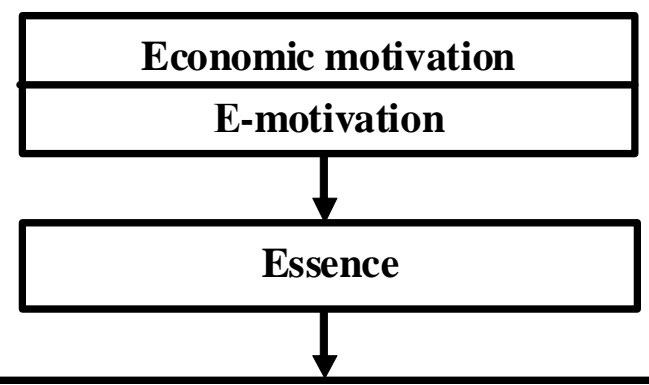

Final orientation or main aim of certain business activity, answers the question what for production, selling and consumption of goods are done

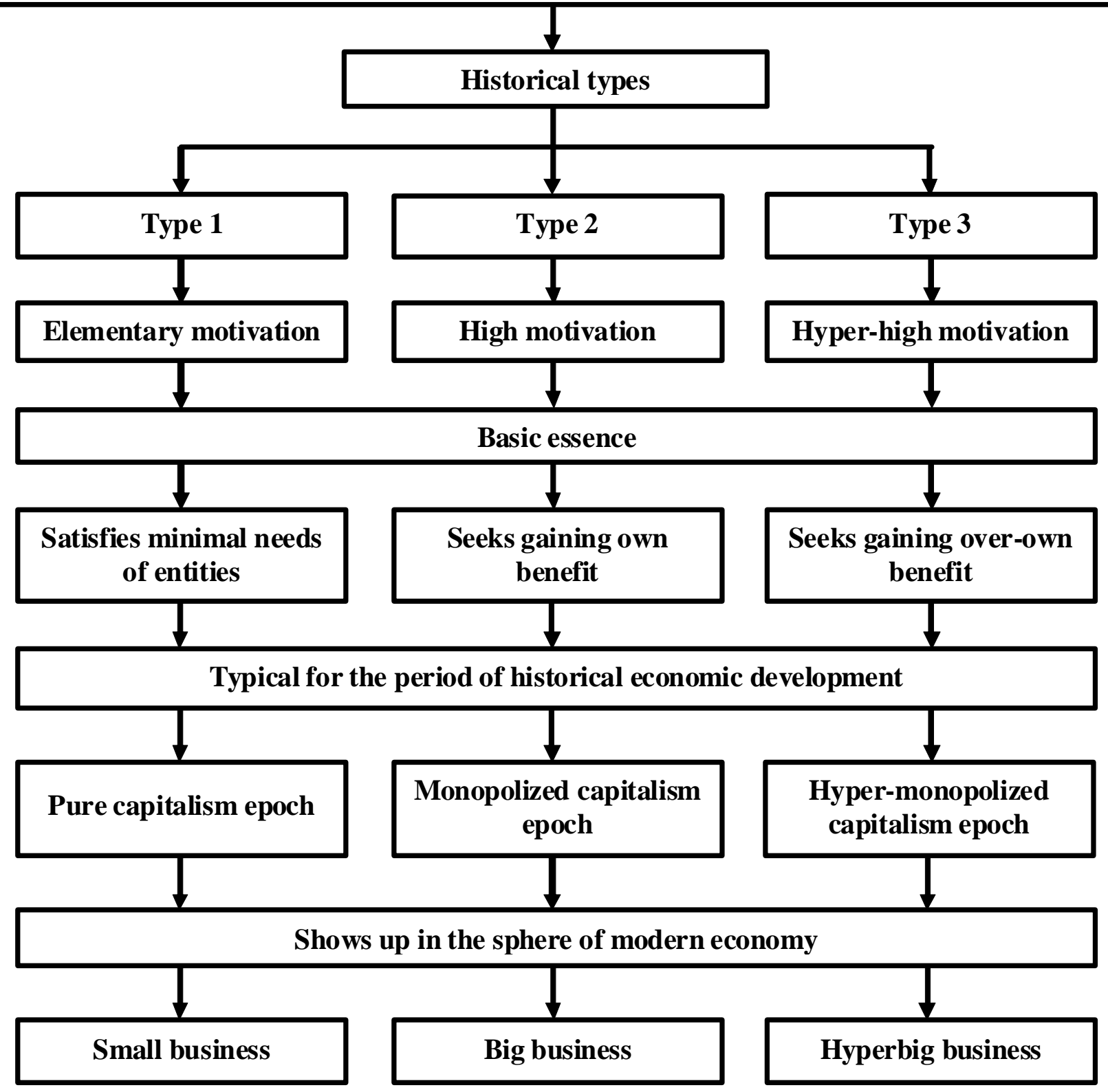

Fig. 1. Main historical types of economic motivation of business entities 


\section{G. I. Bashnyanyn}

This economic motivation also changes with the course of historical and economic development. Its careful study in this historical context gives us reason to single out at least three types of such economic motivation (see fig. 2).

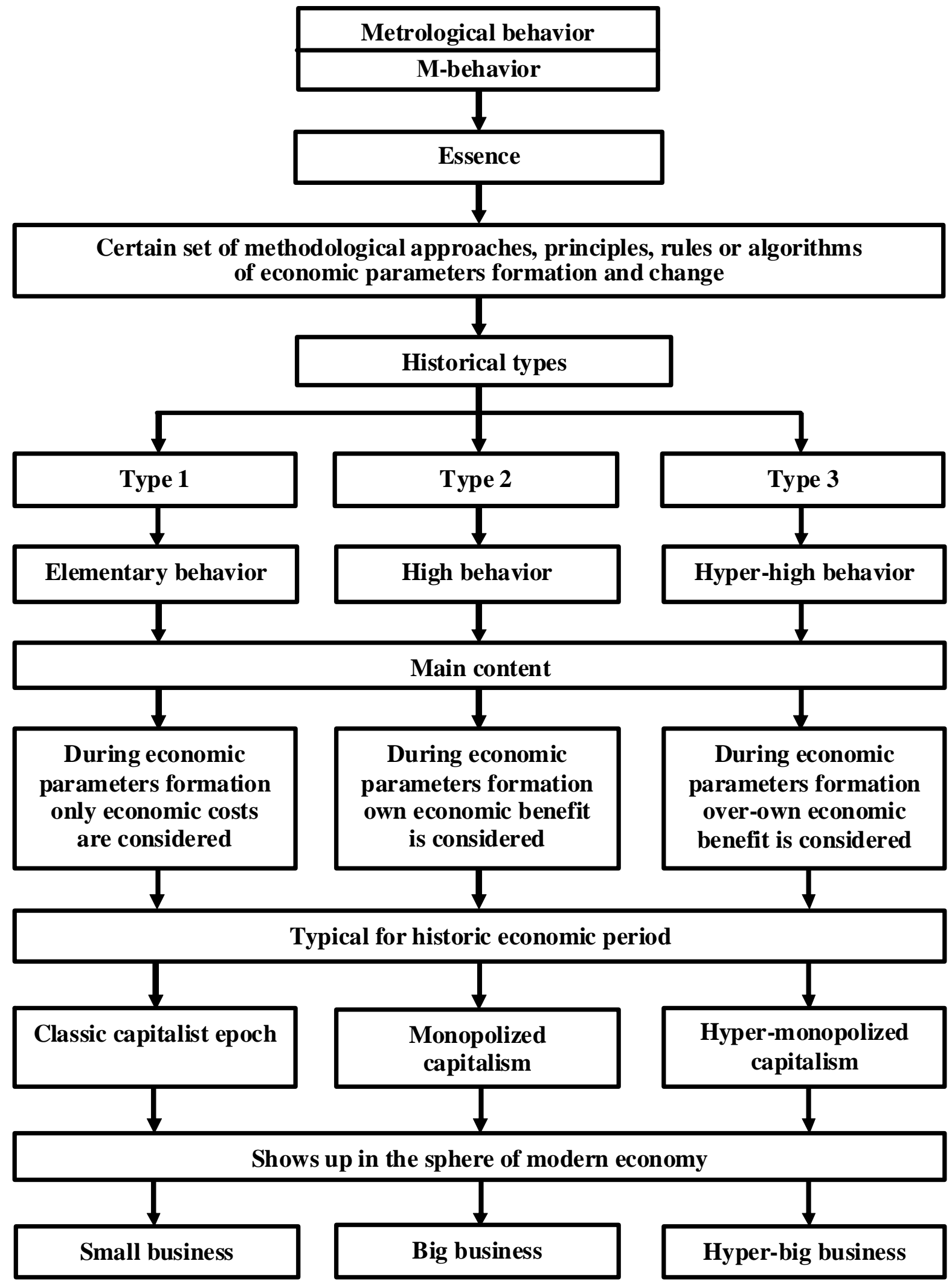

Fig. 2. Main historical types of metrological behavior of economic entities 
Type 1 is an elementary economic motivation (EE-motivation), typical for businesses in a free competitive period of historic economic development in an era of small (or simple) economic systems dominance. In the field of small business entities do not set a higher goal than to satisfy their minimum economic (or socio-economic) needs. This is the essence of elementary economic motivation. In such conditions entities do not aim at getting any economic benefit. The criterion of economic benefit is not yet urgent. The historical era that did not bring economic benefit to the forefront as the primary criterion, or driving force of economic development, was called by K. Marx simple commodity production. This era of simple commodity production continued chronologically (in the modern world's most developed countries) until the XV-XVI centuries, so it can be called protocapitalistic (or pre-capitalistic). However, if it were relevant only in the historical context, there would not be a necessity to research it in modern times. But it is researched. Small commodity production sector or small businesses remain today a separate segment of national economy. It is in this area that the given type of economic motivation dominates, when economic entities do not go beyond minimal satisfaction of their own needs. In this segment of economy the economic benefit has not yet become the major criterion, or driving force of the economic development. Further we show that the mentioned type of economic motivation determines the corresponding type of metrological behavior of entities that form the basic economic parameters by strictly determined and usually the most primitive rules.

Type 2 is high economic motivation (HEmotivation), in which the entities conduct economic activities not only to meet their minimum needs but also to get certain economic benefit. The criterion of economic benefit already comes to the forefront. However, the lower criteria of own minimum needs satisfaction is not eliminated completely, and begins to perform a supporting role or function. Historically, economy enters this criterion dominance zone in the period of emerging monopolized economic systems. It is then that the economic activity begins to be associated with the economic benefit, i.e. any economically beneficial economic activity is considered as economic activity. Thus, truly "economic substance", begins its "life" then and only then when the economic benefit comes to the forefront of any economic activity, to be exact, it is not economic benefit but rather one's own benefit (benefit for the company, though in this early period of modern economic development this benefit is almost always economic benefit; it transforms in the social or socio-economic or socio-spiritual benefit in the next period of economic historical development).

Karl Marx was the first to notice and theoretically fix this significant economic principle in "The Capital". However, he made one "small" (but methodologically essential) mistake he identified the principle of economic benefit as a fundamental principle of economic development and economic functioning with the bourgeois, or capitalist, principle. He thought that only the bourgeoisie as a class can, in its economic activities, be guided by the principle of economic benefit, and as soon as the proletariat removes the bourgeoisie in a revolutionary way, history "will reverse" immediately to the lower principle - that of meeting the minimum needs of its members. This principle of economic development (it is more correct to call it the principle of proto-economic development) was qualified by K.Marx as the natural one that is conditioned by the historical process of economic development and the principle of economic benefit was qualified as a bourgeois one that is deformed. We call this approach and such understanding of economic development motivation retrograded.

The principle of economic benefit or benefit in general is not a bourgeois one, although at some stage it acts as bourgeois. It is, without doubt, the most efficient economic principle, the first great principle of free economic development. Since the time when it begins to dominate, the economy leaves the proto-economic state of development and enters into an era of true development, actually becoming the economy in its modern sense. Hence, to fight against the principle of economic benefit, as Marxists did and continue doing now, means to fight against economic development, strive to "return the economy back" into its proto-economic condition. However, class ideological conclusions, arising from the general characteristics of the principle of economic benefit, are not major for us. More important is to find and theoretically fix metrological consequences of economic motivation principle emergence on the historical scene. 


\section{G. I. Bashnyanyn}

Type 3. With the appearance of the principle of benefit the progress in changing the structure of economic motivation of businesses does not end. In the post-capitalistic (current) period of historical economic development there gradually appears even higher principle of economic motivation, we conditionally qualify it as the hyper-high one (see fig. 1). It corresponds to hyper-high economic motivation, which means that certain entities conduct certain business activities not only to meet their minimum needs or get certain benefit or for their own benefit, but for over-own benefit to meet the specific needs of society as a whole or for the benefit of a certain industry or field of economy. This hyper-high motivation of business gradually began to come to the foreground in the second half of the twentieth century (in the most developed economies of modern world, or in the so-called traditional past capitalism). For its actual formation the process of capital integration had to move beyond big business and enter the zone of hyperbig business. It is such extremely big business that is the form of economy where the subjects stop putting their own benefit in the first place.

Sometimes for them the benefit, which, at first, is not necessarily their own but is the benefit to other businesses has sometimes much more economic significance, we call it over-own or hyper-real benefit; secondly, it is not necessarily purely economic, but may be socio-economic or even social and spiritual, so it can be qualified as over-economic (not only economic but also social or socio-spiritual) benefit; thirdly, it does not necessarily have a static nature, i.e. act as a static benefit, and may be remote in time to a particular economic period, it can also be defined as overstatic benefit).

An extremely big businessman (or an extremely big monopolist, as K. Marx would put it) no longer wants to receive only that benefit, which is, of purely his own, purely economic, and purely static character. Horizons and criteria of his business are significantly expanding, and due to this, a new higher-quality spiral of economic progress occurs. If in medium or large economies (actually capitalistic) economic progress was made through implementing the principle of own or real benefit, now, in an era of ever larger business when there appeared hyper-big economic systems (the definition given by the author - G.B.), the economy develops on the basis of this hyper-high economic motivation. The more different economic systems are focused on the practical implementation of the highest motivational approach, the more sophisticated and developed they become. From the moment when the motivational approach entered the historical arena, economic development transformed from capitalist into post-capitalist. Truly economic is not only economically profitable for this company, but also for the economy as a whole and not only at this time, but in the long run. The economic motivation of economic activity in the recent historical era becomes long-term in terms of time and functional level and much more colorful in terms of formation (not only "economic" but also "social" and "socio-spiritual" color of objective economic activity are taken account of).

This change (being already the second one in order) of economic motivation of economic activity becomes the determining factor of all socio-economic development. It marks another revolution in the economic practice. In modern economic literature it is sufficiently covered, but today almost no analysis of its metrological (measurement) consequences is being conducted. That is why we set a goal to make a deep scientific analysis of the metrological side of the problem, or the described above change in the nature of economic motivation of economic entities. Meanwhile, the hyper-real economic motivation is especially strongly manifested in the hyper-big sector of the national economy, the sector of hyperbig business.

To sum up, the transition from elementary to high and hyper-high (real and hyper-real) economic motivation of economic activity is influenced by fundamental factors such as consolidation of capital. The general trend is as follows: the more capital an economic entity possesses, the more perfect and progressive its economic motivation, its motivation policy will be. Economic motivation is the most perfect not in conditions of perfectly competitive market, as the liberal economists believe, but in conditions of the market that is the most "distant" from the pure competition. Economic progress of various economic systems is growing with their scales growth. Gradual transition from extra-small, small or medium economic systems to large or hyper-big ones simultaneously defines the primary direction of social and economic progress, the change of elementary motivation to high or hyper high. 
Liberal economists (both of classical and modern postclassical branches of economic science) turned the socio-economic development "upside down". They continue to believe the pure perfect market to be the most progressive, it being in reality the least progressive of all currently existing types of markets, i.e., they idealize small or extra-small economic systems. Only when the market is transformed from the pure perfect into the imperfect (being monopolized in this or that way, i.e. big or hyper-big) market, it progresses, changing its economic motivation from elementary to high or hyper-high. This theoretical conclusion has a very great methodological importance for the research of operation and development of metrological systems in the economy, a special study of which the author began in the early 80 s and today actively and purposefully continues to analyze in his works.

\section{About the main historical types of metrological behavior of economic entities \\ As has already been noted, economic motivation and its nature (and its historical transformation) determines metrological behavior of economic entities. It also transforms historically, moving from a lower to a higher degree of development. It is understood as a set of} methodological approaches, principles, rules or algorithms to the formation and change of the key economic parameters. Since among these parameters there are primary (the formation of value, for example) and secondary (all those derived from the value or price), then the metrological behavior of economic entities can be differentiated as primary, basic, major, and secondary. The first should be explored within the general economic metrology, and the second within the special one. In this part of our analysis we focus on the issues of general economic metrology; we are primarily interested in the part of metrological behavior of economic entities limited to the formation of such parameter as the value or price. The author began the study of the nature of metrological behavior of economic entities in the 80s. Steadily and consistently following the historical approach to the analysis of metrological issues, we managed, on its basis and in its methodological paradigm, to single out a number of historical types of metrological behavior of economic entities, each of which is the subjective environment, in which the appropriate type of economic measurement is formed (fig. 2).

Type 1 is elementary, or lower degree of development of the economic entities metrological behavior, which prevailed in the classical capitalist era (in the era of simple commodity production, according to Maxist periodization of economic development) and today it is saved in the purest form in small business (in small economic systems, in our terminology - G.B.). This elementary metrological behavior of economic entities means that the latter in determining, for example, the value - price or economic evaluation (in the absence of inflation, and when economic evaluation stands in cash, these three concepts, or measurement forms, act as de facto identical) take into account, first of all, economic costs. The greater they are in the production or consumption of certain goods, the greater value these goods have.

Although elementary metrological behavior of economic entities was historically considered to be primary, it is still preserved in those sectors of the economy in which small business dominates. Thus, elementary metrological behavior is the measurement ideology of the poorest group of businessmen, and so it appears to be the most left during political assessment (more precisely, "the most left" among the "right" ideologies of measurement). Small businesses do not aim at getting any economic benefit from their economic activities. It is more important for them, as we have already pointed out, to fully meet their minimum material and economic needs.

So, small businesses want, with an objective necessity, to produce or consume the goods the value of which gives the opportunity, on the one hand, to recover all costs of economic resources (including their own entrepreneurial skills), and on the other hand, to most fully meet their minimum needs. That is why they will "choose" for doing business (consciously or unconsciously) only those "niches" in which goods or services have only nominal value. Such goods, firstly, must be technologically simple, secondly, perfectly competitive, thirdly, their production or consumption should be possible with small capital (their capital capacity must be the lowest). Such goods with very low capital capacity give, firstly, very small, and secondly, almost sudden money income, which almost always goes to meet a particular entity's own needs. 


\section{G. I. Bashnyanyn}

Type 2 is a high or higher metrological behavior of economic entities, which becomes real in the sphere of big business or in the era of monopolized capitalism (the era of imperfect competition). In its conditions, economic entities assess the value of goods and services (or other economic processes and economic activities) not only on the basis of their economic costs (that is still typical today for the elementary metrological behavior) (the goods accompanied by more economic costs are "attributed" or "imposed" higher or greater value), but also on the basis of their effectiveness, economic benefit or economic effect that accompany production or consumption of certain goods. More cost-effective goods and services have higher real value, are worth more.

At high or higher type of economic entities metrological behavior the criterion of economic benefit (more precisely, not only economic but also own benefit, such as the one being formed at the moment, i.e. sudden benefit) comes to the foreground. Cost-effective goods are treated as goods which possess conventionally greater value, they cost more (metrological estimation in the economic sphere of the economy and in the society as a whole always has conventional, more exactly, formal nature since it characterizes the measure of importance of goods and services and that is why it concerns only a formal part of the economic matter movement; it is similar to economics itself that is a formal rather than real science, thus being close to the sciences of mathematic cycle). This metrological behavior of business entities is projected on other (but for economic) structures of the society. In its system more beneficial processes receive higher evaluation; this evaluation can be both economic and beyond economic. But beyond the economic sphere the value already exists as a rating, or as a non-economic value (social value, social and ethical value etc.). In this part of work we put an economic value as a major form of metrological outcome in the economic sphere of the society.

High (or higher) metrological behavior of economic entities historically was formed in the era of classical capitalist structures transformation into monopolized capitalist systems, or posttraditional capitalist economic systems, big capital (big business) being the material basis for their operation and development. .And it was dominant throughout the whole postclassical stage of capitalist development, i.e. until the end of the first half of the twentieth century (in modern most developed countries of the world). In conditions of post-traditional capitalism all or almost all economic processes, economic actions or economic goods and services are evaluated (i.e., are reflected metrologically) almost exclusively on the basis of their benefit, which is, firstly, economic, secondly, own, serving as a benefit for a particular company, thirdly, static which can be received practically immediately, at the moment of performing certain economic activities or economic spending. Such a businessman (a big businessman) is not interested in any other benefit (it being noneconomic, non-microeconomic, not his/her own and non-static), he/she ignores it, so he/she pays attention only to economic projects or economic decisions that can bring him immediate benefit, necessarily "in money" but not in any other goods or services.

This metrological behavior is a step forward compared to the elementary one, because it no longer limits the measurement activity to accounting only economic costs, it is more progressive. But it also has some disadvantages, in particular, it has a purely economic nature (uneconomic part of benefit or effect is not included in the evaluation activity), and secondly, it considers only local benefit, i.e. a competitive advantage for a firm (own benefit); thirdly, it also takes into account only the static benefit, i.e. the one that is formed immediately at the time of performing certain economic activities (the socalled zero time lag). Economic, local and static natures of higher metrological behaviour are essential to its further transformation into a still higher one, which we qualify as hyper-high.

Type 3. The process of escalation, or transformation of high metrological behavior into hyper-high (a third historical and functional type) began in the most developed countries in the second half of the twentieth century, and have not yet ended. Now at the beginning of the XXI century we can observe the hyper-high metrological behavior that was scientifically described by the author in $80 \mathrm{~s}$, and further investigated in 90s. The hyper-high metrological behavior is especially clearly manifested in hyperbig business or hyper-big or hyper complex economic systems. In such a business system, 
economic entities, purely metrologically, begin to act more rationally, more perfectly, more precisely than, say, in big business. For them, their own benefit ceases to be the main criterion of value of certain goods (moreover, it being non-economic and static).They are more targeting not only and not so much their own benefit (of economic and static order), but more the over-own benefit. The latter is not only static but dynamic, can be formed and implemented not only at this time, but in the distant period, it is also not only necessarily just economic but also social and economic or even social and spiritual. Here, for the first time in history the economic entities begin to act not only economically, not purely statistically, and not purely micro-economically, but also socioeconomically, dynamically and increasingly globally. Local benefit (only for this very company) ceases to dominate, but retains the role of the partial formation parameter of a new (more precisely, contemporary) metrological behavior.

If the traditional (elementary) metrological behavior of economic entities was sufficiently investigated in classical and modern neoclassical economic literature, and the new metrological behavior - in the classical literature (especially Marxist, but only as a context), the newest metrological behavior, peculiar to modern era of the global economic development, is almost totally unexplored. The author started its study in 80-90s, making a significant contribution to its research. And so a new or modern turn in the development of econometrics should be based primarily on a preliminary analysis of modern metrological behavior that is characteristic for the sphere of big business. Its detailed and careful study gave us reason to conclude that the newest (hyper-high) metrological behavior is based on the account of, firstly, not only micro- but also global (macro-, national economic etc.) effect or benefit; secondly, not only static but dynamic effect or benefit, thirdly, not only economic but also socio-economic or socio-spiritual effect or benefit. Hyper-big capital embodied in certain economic entities is, firstly, more farsighted in terms of time; secondly, more farsighted in terms of functional levels, and thirdly, more farsighted terms of formation. In "metrological terms" it sees farther and higher, and therefore the results of measurements at this metrological behavior are more accurate, in metrological terms. From the point of view of politics, they are more rightwing If a small business leads "a classical right" policy, then large or very large business conducts hyper-right or over-right policy, which metrologically is the most accurate and most advanced policy.

This conclusion, of course, will shock the representatives of the "left wing" of the political spectrum of society, but it is objectively conditioned and reflects the real development of business practice in general and such an important part of it as the practice of economic measurement, that is materialized in the corresponding metrological behavior of businesses and transforms in a particular direction in the course of historic and economic development. Such approach and such assessment is presented in metrological work of the author, particularly in those published in 80-90s of the twentieth century.

\section{About fundamental types of economic measurement}

Three types of metrological behavior of economic entities conditioned three types of economic measurement - elementary, or nominal; high, or real; and hyper-high, or hyper-real (or, respectively, traditional, post-traditional, or new and modern) (fig. 3).

Type 1 is elementary, nominal, or traditional economic measurement based on the nominal measurement ideology. Its essence is that in determining (assessing) the value of certain goods and services only economic cost of the basic resources is taken into account. The greater they are, the greater the value (importance) is attributed to (or "imposed on") a particular good. Under the dominance of the nominal ideology of economic measurement the major economic parameters (particularly value) fully reflect the movement or the value of economic costs. Economic costs, thus, serve as some economic measure of all major economic parameters, economic forms or economic processes. For example, the value of any good in full (without any remainder) is reduced to economic costs, i.e., it will always be possible "to decompose" its value (also without any "remainder") into costs of the economic order. 
G. I. Bashnyanyn
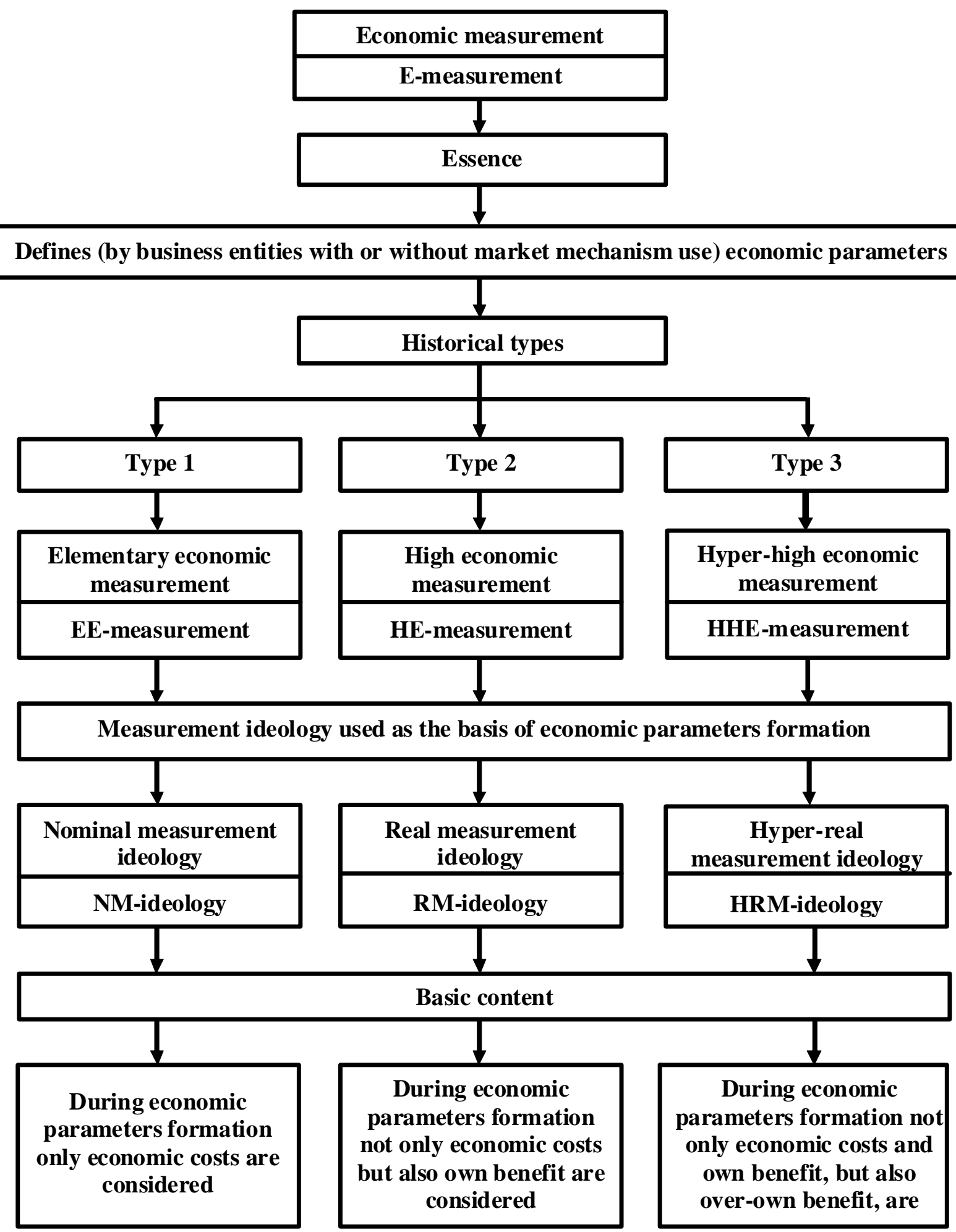

Fig. 3. The most fundamental historical types of economic measurement

To define this or that parameter by its nominal means to make an accurate calculation of economic costs that "represent" this parameter or act as a form of it. Nominal ideology of economic measurement "works" more or less sufficiently under two conditions. First, under the so-called pure competitive market conditions, or pure competitive economic systems in which certain economic activities are more or less "equally effective" that gives equal and rather miserable economic benefit. The latter, under such economic conditions, is not larger (as a rule) than the normal 
profit, the amount of which more or less coincides with the costs of such a specific type of economic resources as entrepreneurial skills. The normal profit practically (both qualitatively and quantitatively) does not differ from wages as a sort of metrological reflection of labor costs, particularly concerning physical labor (as the nominal ideology of economic measurement dominated in the era of traditional labor based on physical labor, and mental labor did not play any significant role). The second case when the nominal ideology and (the corresponding type) economic measurement also dominate, is that when the economy is functioning as an administrative economy. During the rule of administrative economic systems economic measurement can be only elementary, or nominal. This conclusion is confirmed by all the experience of the so-called socialist and later communist construction in the countries of the so-called real socialism (or the socialist camp). In such economies, the economic cost is the measure of all key economic parameters, without "the remainder", i.e., in fact, the only measure that excludes the application of any other measures.

But the deeper cause of the practical existence of the nominal ideology (or the type) of economic measurement is the character of economic motivation of economic entities. Until the economic entities set a higher goal than a minimum satisfaction of their needs, the practice of economic measurement is always carried out according to a nominal scheme that best corresponds to the economic motivation of small business. In the system of such business, economic measurement can only be nominal by the character, or nature. In other words, for the nominal ideology of economic measurement to be practically real, the capital while consolidating should not go beyond a small capital. It is this capital that is, on the one hand, the material basis for the existence of small (hyper-small) economic systems, and, on the other hand, gives rise to the nominal ideology of economic measurement.

From the historical background, it can be noted that the economic dominance of the nominal measurement was most characteristic of the classical capitalist era of modern economic evolution, when the capital was still too small for perfect, more advanced measurement ideology to enter the historical scene. The culture of economic measurement in that historical era was still very low, was at the elementary level, where only economic costs were the criterion or measure of all major economic parameters. The objective "undermining" of this nominal ideology of economic measurement began when the capital evolved beyond small capital, stopped being too small, somewhat concentrated. So, the epoch of post-traditional capitalism (post-capitalist economic systems) formation is the era of "the beginning of moving away" from the nominal ideology and culture of economic measurement. It was this epoch that called to life a new historical type of economic measurement, which we qualify as a real, post-traditional, and actually new.

Type 2 is the real type of economic measurement, which historically replaced the nominal type; it is characterized by the fact that only economic costs stop being the measure of value of certain goods or services; these costs are not eliminated as a measure of value and preserve their role, but already as a separate option. When this or post-traditional (new) type of economic measurement is used, the value of goods is determined by the economic benefit as well, by the degree of efficiency of goods and services. Better goods are regarded as having greater value. We call this ideology of economic measurement, which prevailed in the era of monopolized capitalist development, the real ideology (and the corresponding type of economic measurement based on this metrological ideology could also be qualified as real). However, in this case "real" is understood not in the sense of its price (as it is often done in modern economic literature), but in the meaning of its formation and function when economic magnitudes, whose values or structures also reflect the degree of effectiveness of certain goods or services, certain economic processes and economic activities, represent real economic volumes. Nominal measurement, in its turn, is seen as indifferent to this economic parameter, i.e., it has no relation to the reflection of degree or measure of effectiveness (in the modern economic literature the nominal volumes of economic parameters are their volumes in the so-called real prices, i.e. with the account of the inflation component. This understanding of nominal can also be considered as traditional).

The real ideology of economic measurement is more perfect than the nominal one; it marks a 


\section{G. I. Bashnyanyn}

new stage in the development and formation of the economic measurement culture. Using the outlined real approaches to measurement of economic parameters as the basis, we raise the general culture of economic measurement to a higher level, make its results more precise and perfect. Real ideology of economic measurement is implemented through the corresponding mechanism of market pricing. For the market and market structures to transform nominal economic measurement into the real one, the market should transform from the perfectly competitive into the imperfect (i.e. monopolistic) market, which possesses the methods and mechanisms that allow, due to the combined effect, transforming nominal economic measurement into the real, higher and more perfect. However, the monopolistic market is formed by the same economic factor as the corresponding metrological behavior of economic entities - due to the increase of the capital scale. The scale of capital, or the total money income, which the economic entity possess or have at their disposal, is the driving force that transforms not only the nature of the market but also the corresponding metrological behavior; and at the same time the type or nature of economic measurement is transformed too. Since the beginning of the formation and development of large economic systems (based on large capital) metrological behavior of economic entities has changed drastically. It is based not on the nominal but on the real ideology defining the basic economic parameters which no longer are indifferent to the effectiveness of certain goods or certain actions. This interpretation of the real ideology of economic measurement (and the appropriate type of measurement based on this ideology) for the first time in modern economic literature was suggested by the author in the late $80 \mathrm{~s}$, and in some publications of the first half of 90s. Later it has been developed in our metrological works in the second half of 90s in which we clearly and unambiguously connected the real approach to economic measurement with the so-called effect-based one. We recommend to determine the real volume of certain economic parameters with the account of the effect (economic effect), which is formed in the process of undertaking respective economic activities. Goods with greater effect are recommended to be regarded as having greater value (what we call the real value).
However, the real economic ideology of measurement has several shortcomings, is not perfect, if to evaluate it, say, by the degree of economic progress or the accuracy of measurement results. Its main drawbacks are: firstly, it takes into account only its own effect in the real volumes of certain parameters, which some competitive firm receives. The part of economic effect formed outside the firm, is not considered when using the traditional approaches to real economic measurement. Sometimes this part of effect is quite significant and ignoring it means a significant reduction in the accuracy of economic measurement. When using the real ideology of economic measurement we consider (due to the corresponding metrological behavior and through appropriate market mechanism) only the local effect, and the real ideology of economic measurement has the local character. Secondly, based on the practical use of the real ideology of economic measurement in real volumes of various economic parameters, we present only the net economic effect. As it is known, the integral effect in any modern economic activities is only rarely reduced only to the economic part. It more or less represents socio-economic, social and spiritual parts, which are not covered and displayed by this type of measurement ideology. Finally, the third drawback in the real ideology of economic measurement is as follows: on its basis we can detect and display only that part of integral effect, which is formed at the moment, suddenly, almost simultaneously with certain economic activities.

The above disadvantages of real ideology and economic measurement technology often go unnoticed in the modern economic literature. It (especially in its neoclassical branch) considers that competitive market is the most perfect and most accurate economic measurement tool. We show here that this is not so. On the one hand, a perfectly competitive market is indeed a more accurate measurement mechanism than, say, its historical predecessor (the market of the simple commodity production era). It is the first type of market that really starts to take into account such a parameter as effect, benefit in the formation of economic variables. This market is oriented towards efficient economic activities. This is its undoubted advantage over the proto-market that prevailed in the pre-capitalist era. However, on the other hand, the measure of reflection of the 
"effect" parameter is obviously incomplete, insufficient. Only in the objective limits of big capital, which "selects" for production those goods and services that possess only economic, micro-economic (local) and only static effects, the perfectly competitive market is a more or less accurate measurement tool. Outside, in the zone of hyper-big capital, which draws in its area those goods and services that possess not only local but also global, not only economic but also socio-economic or socio-spiritual, not just static but the dynamic effects, the perfect competitive market is inaccurate and imperfect. Such a conclusion was first made by the author in the late 80 s.

Type 3. Imperfection and inaccuracy of the perfectly competitive market are eliminated in the course of its historical transformation into the monopolistic, especially hyper-monopolistic market, which operates and develops on the basis of practical use of the hyper-real ideology of economic measurement, within which the appropriate type of economic measurement is formed (through the appropriate metrological behavior of entities), which we qualify as modern, hyper-high, or hyper-real. The hypermonopolistic market, formation of which took place in the second half of the twentieth century (in today's most developed countries or the countries of the so-called traditional capitalism) is the market mechanism within which the hyper-real (hyperhigh, modern) ideology of economic measurement became true. According to its terms, economic entities (usually hyper-big businessmen) begin to determine the real value of goods not only in terms of economic costs, not only in terms of real effect (under real effect we understand, firstly, the economic effect without the part that has socioeconomic or socio-spiritual nature, and secondly, micro-economic effect only as a quite general level of local or global, otherwise, the integral effect and thirdly, the static effect as that part of the functional-dynamic effect, which is formed at a given time, actually or virtually simultaneously with certain economic actions), but also in terms of the hyper-real effect (the term is also actively used in our previous metrological works), the effect, which is formed not only at this moment of time, but in the nearest or somewhat longer perspective, which acts not only as its own (micro-economic) benefit but also as the benefit of the global order, which has not only economic nature but also socioeconomic or socio-spiritual one.

The hyper-high (hyper-real) ideology of economic measurement that can be practically implemented only in conditions of the hypermonopolistic (hyper-monopolized) market is an order higher than high, or, as we marked it earlier, the post-traditional ideology, which is most clearly manifested in conditions of the monopolized market. Thus, the historical transition from the perfectly competitive market to the less perfectly competitive market (hyper-competitive, quazicompetitive, hypo-competitive, absolutely imperfect competitive market) marks and practically reflects the ideology of economic measurement. It gradually transforms from high or post-traditional (real, in our terminology) into hyper-real, newest, hyper-high, most common for modern economic era (which chronologically covers the second half of the XX century and the beginning of the XXI century). And this fundamental historical transformation of the perfectly competitive market with its own state into the monopolistic and hyper-monopolistic market, on the other hand, is not a manifestation of economic recession, how it is considered in modern liberal (neoclassical) literature, but a manifestation of economic progress.

From purely metrological position, this historic transition to a new stage of market economy means at the same time the increase of the economic measurement accuracy. The hyperhigh, or hyper-real ideology of economic measurement is based on the account of the full effect, or full benefit (in functional-level, functionalformation, and functional-dynamic respects) while in fact the real ideology takes into account this benefit or effect in the process of certain economic parameters determination only partially, only to some extent. This gives grounds to qualifying hyper-real measurement as general, and real or even nominal - as a special case of the first. The hyper-real measuring ideology is the product of historical development of the real ideology that plays the same role in respect to the nominal ideology. There is or there should be a certain historical relevance between them, which in the quantitative form can be reflected as metrological relevance.

Therefore, examining the structure and features of hyper-real measuring ideology (and 


\section{G. I. Bashnyanyn}

technology), we should be able to search for some coefficients of conduction or reduction (transformation) of one type of measurement into another. All the three basic types of economic measurement - nominal, real, and hyper-real are both monistic in its basis (all of them can be reduced to a nominal measurement as the historical basis, or historical pre-condition) and pluralistic, diverse, heterogeneous. A heterogeneous element in them is an effect or benefit of the corresponding type (hyper-real or real effect or benefit). It is this element that is the historical increase of the economic matter, more precisely, a reflection of this historical increase.

Conclusions. As a result of scientific analysis of both historical and functional practice of economic measurement the author reached a fundamental conclusion that the character (nature, type) of economic measurement is determined by such economic factors as, firstly, the historical type of economic motivation of economic entities and, secondly, by the historical type of their metrological behavior. In the basis of these two factors there is even a more fundamental factor the functions of which are fulfilled by capital scales, system's real scales, in which a certain type of economic measurement is implemented. The larger the capital scales are in a certain economic system under study, the more perfect, and, consequently, the more accurate an appropriate type of economic measurement is. Three types of both economic measurement and metrological systems in economics are: nominal - real - superreal types of measurement; and appropriate metrological systems in economics correspond to three fundamental scale classes of economic systems - small, large and super-large. Nominal systems of metrological class are formed in the environment of small capital, small business, but real and super-real - in the environment of, accordingly, big and super-big capital and business. Capital scales, as the author of the article concludes, change the "length", "scale", "measure", so to say, both of the main "economic ruler", or the main "econometer", the functions of which are fulfilled by money and of the main measurement result - the economic value and the economic price of traditional goods. As system's real scales grow (capital real scale), which are determined by the income scheme rather than costs scheme, like, for example, nominal scales, the main "econometer' (money, "economic money") "shrinks", reduces its "length" as a kind of "economic ruler" and the value and price of goods, by contrast, "lengthens" "increases its length" in a somewhat geometric style. Thus, capital scales (but real, not nominal) play a role in the practice of economic measurement as a kind of "economic gravity", they, like the power of "gravitation" in the natural world, also "lengthen" or "shorten" space and time, but already "economic space" and "economic time", which are "embodied", on the one hand, in the real value of money as the main "economic ruler" and, on the other hand, in the real value and real price of goods as the main economic measurement result in economic life. This article is namely dedicated to the justification of this fundamental theoretical conclusion, which proves that, firstly, the metrological behavior of economic entities is derived from their motivational behavior, and, secondly, in the basis of changing the nature of economic measurement practice there is such a more fundamental factor as capital real scales, in the "environment" of which this or that type of both economic measurement and metrological systems in general as somehow organized practice of economic measurement is formed.

\section{References}

1. Bashnyanyn G. I. About new interpretation of national-economic formation model and problem of ownership / G. I. Bashnyanyn // Sotsialisticheskiy trud. - 1989. - № 11. - 75 pp.

2. Bashnyanyn G. I. Economic measurement of intellectual goods / G. I. Bashnyanyn. P. 2. - Lviv: LTEI, 1990. - 45 pp.

3. Bashnyanyn G. I. Economic measurement of intellectual goods / G. I. Bashnyanyn. P. 3. - Lviv: LTEI, 1990. - 35 pp.

4. Bashnyanyn G. I. Metrological economic systems: introduction into the general theory and methodology of formation of economic parameters / G. I. Bashnyanyn. - Lviv: Publishing house of Lviv Commercial Academy, 2012. - 1152 pp. 